\title{
Emergent and delayed hybrid external fixation management of tibial pilon fractures: A multicentric retrospective analysis of 80 patients
}

Giuseppe Rollo ${ }^{1}$, Marco Filipponi ${ }^{1}$, Paolo Pichierri ${ }^{1}$, Valentina Russi ${ }^{2}$, Lorenzo Nalbone ${ }^{2}$, Michele D’Arienzo ${ }^{2}$, Sara Cavalera ${ }^{3}$, Gianfranco Corina ${ }^{4}$, Michele Bisaccia ${ }^{5}$, Luigi Meccariello ${ }^{1 \bowtie}$

${ }^{1}$ Departement of Orthopedics and Traumatology, Vito Fazzi Hospital, Lecce, Italy

${ }^{2}$ Orthopaedics and Traumatology Clinic, the University of Palermo Hospital 'Paolo Giaccone', Palermo, Italy

${ }^{3}$ Departement of Orthopedics and Traumatology, University Hospital "Policlinico di Modena", Modena, Italy

${ }^{4}$ Departement of Orthopedics and Traumatology, Di Summa - Perrino Hospital, Brindisi, Italy

${ }^{5}$ Division of Orthopedics and Trauma Surgery, University of Perugia, S. Maria della Misericordia Hospital, Perugia, Italy

\section{ARTICLE INFO}

Article history:

Received 21 April 2017

Received in revised form 21 May 2017

Accepted 26 May 2017

Available online 1 July 2017

Keywords:

Tibial Pilon

External fixation

Hybrid external fixation

Outcomes

Open fracture

Soft tissue Injury

\section{ABSTRACT}

Objective: To report our experience with the hybrid external fixator in emergency. Methods:We assessed 80 cases of pilon fracture treated with the external fixator during the period of January 2009 and December 2016:55 men (69\%) and 30 women (33\%) with a mean age of 40 years (range between 16 and 70 ). About 45 occurred as isolated trauma, 35 instead were politrauma. Each patient underwent standard radiographic examination and a CT examination. There were 28 open fractures (35\%), (Gustilo type 1, 2 and 3) while closed fractures showed soft tissue involvement of various grade (2-3 Tscherne classification). In all cases, the external fixation, sometimes associated with other reduction and synthesis techniques, was used. The timing of surgery was dictated by the condition of the soft tissues. For clinical evaluation, the Mazur score with mean follow-ups at 12 months was utilized. Results: The final range of ankle motion was 15 dorsal and 10 plantar flexion. In about 80 cases there was an average Mazur score of 83 . The mean score was 90 ; in open fractures 85 to 72 . Radiographic healing of fractures in 60 patients occurred in 120 days (mean 105 days), at the time when the external fixator was removed. Conclusion: Pilon fractures are complex and often present complications; the definitive treatment, in emergency or delayed, with hybrid external fixator permits a stable synthesis with minimal soft tissue damage. Weight bearing maybe allowed early and functional recovery is generally good.

\section{Introducion}

Distal tibia fractures, located within $5 \mathrm{~cm}$ from the articular rime, are about $1 \%$ of all lower limb fractures and about $5 \%$ to $7 \%$ of the tibial fractures [1,2]. Although relatively rare, these injuries, currently on the rise, pose a serious challenge to the orthopedic surgeon because the muscular layer is not adequate, the pilon is poorly

${ }^{\square}$ Corresponding author: Luigi Meccariello, MD, Department of Orthopedics and Traumatology, Vito Fazzi Hospital, Via Ada Cudazzo, Block: A- Floor:V, Lecce, Italy E-mail: drlordmec@gmail.com

Phone: +393299419574

Fax:+390823713864 vascularized, and often there is a comminution of the fragments and joint involvement. Most of these fractures are secondary to a highenergy trauma, such as falling from a height, traffic accidents with metaphyseal sinking, crushing of soft tissues or even exposure of the fracture. Low-energy trauma (eg. Skiing accidents), instead, cause spiral (torsion) fractures, less complicated with modest soft tissue damage [3-5].

They are often associated with open wounds or serious closed soft tissue involvement, with metaphyseal or articular comminution or shaft extension [5].

A careful clinical examination is essential because these fractures are accompanied by marked tumefaction, blistering, swelling, deformity and crepitus around the ankle. The examination shall 
include motor and sensory function of the foot structures, and, in addition, the search for any sign of compartment syndrome [5].

For a rapid total compression of the fracture, the entity of the open fractures must be assessed according to the Gustilo and Anderson classification [6], while the extent of soft tissue damage should be graded according to the Tscherne classification [7].

Severe compound fractures or fracture-dislocations have to undergo to immediate and temporary stabilization [5]. The treatment of pilon fractures is very complex and is based on the evaluation of diagnostic imaging.

CT with two and three-dimensional reconstruction was used to accurately determine the direction of the rime of fracture, the extent and articular fragments displacement. The CT therefore constitutes an important help in the decision making process and the type of surgical incision to be made (Figure 1).

The purpose, then, of this study was to evaluate the effectiveness of the definitive treatment, in emergency or delayed of the pilon fractures using the hybrid external fixator, in a sample of 80 patients over a period of time sufficient to assess the outcomes of this treatment.

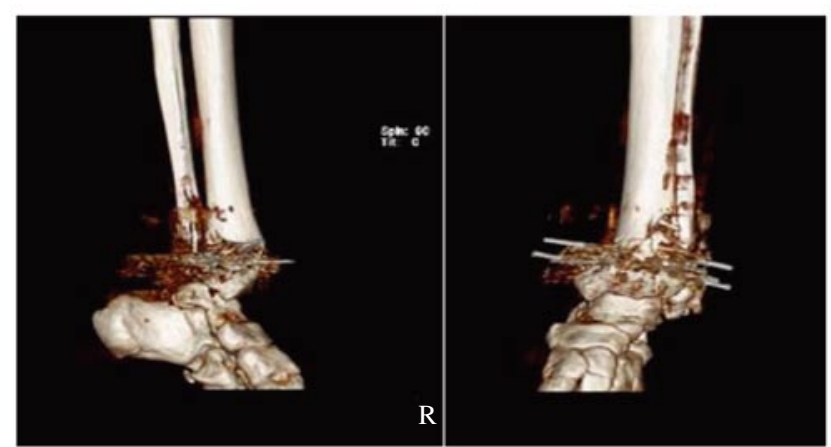

Figure 1. CT images about a complex fracture of Tibial Pilon.

\section{Materials and methods}

We assessed 80 cases of pilon fractures treated with hybrid external fixator in the hospital "Vito Fazzi" of Lecce, the hospital "Paolo Giaccone" in Palermo, "Di Summa-Perrino" Hospital of Brindisi and University Hospital of Perugia during a period of January 2009 and October 2016: 55 men (69\%) and 30 women (33\%) with a mean age of 40 years (range between 16 and 70 years).

About 45 occurred as isolated trauma, 35 instead were politrauma. For an accurate assessment of the fracture, each patient underwent AP and LL radiographs, followed by CT scans.

There were 28 open fractures (35\%), (Gustilo type 1, 2 and 3) while closed fractures had soft tissue involvement of various grade (2-3 Tscherne classification) [6,7].

Fractures were classified according to the AO/OTA [8]: 14 Type A, (17.5 \%, A1:3, A2: 7; A3: 4) 26 Type B (32.5\%, B1:4, B2: 13, B3: 9), and 40 Type C (50\%, C1: 21, C2: 8; C3:11). Twenty-eight (35\%) open fractures were classified as type 1 (10), type 2 (9) or 3 (9) Gustilo - Anderson, and $52(65 \%)$ closed fractures were classified as type 1 (24), type 2 (10) or 3 (19) Tscherne.

All cases required external fixation possibly associated with other reduction and synthesis techniques. The timing of surgery was dictated by the condition of the soft tissues.

On 11 patients (14\%) surgery was performed in emergency and treated with external fixation; on 9 patients $(11 \%)$ instead, a final external fixator was mounted in emergency.

The remaining 60 patients $(75 \%)$, after the trauma, were treated with trans-calcaneal traction and venous drainage of the limb, in order to delay surgery for about 7 days until complete healing of the blisters and restitution ad integrum of the soft tissues involved were achieved, (range 1-15 days) (Figure 2).

\section{Surgical methods}

= Temporanely external fixation

= Definitive external fixation

m Transcalcaneal $\mathrm{K}$ wire and after defenitive external fixation

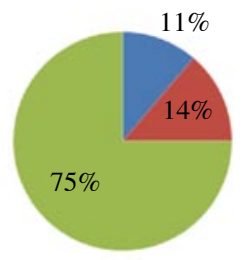

Figure 2. Types of surgery.

All patients, after surgery, underwent antibiotic therapy and prophylaxis with low molecular weight heparin (LMWH).

The chosen criteria to evaluate the group during clinical and radiological follow-ups were the following: The Mazur score [9] was used for clinical evaluation, (the maximum score was 100; a value higher than 92 was considered very good, 82-92 good, 65-86 fair,< 65 was considered insufficient). The XR standards were used to assess bone healing. At last, possible complications were evaluated. Follow-ups were performed with clinical and radiographic controls at 1 month, 3 months, 6 months and 12 months and annually thereafter. The assessment endpoint was set at 12 month follow-ups.

\subsection{Surgical notes}

The surgery was performed with the patient in supine position and the affected limb in transcalcaneal traction. Under luminance control, an external fixator was mounted with three Kirschner wires placed distally to the fracture site, at an angle of 40 and respecting the "Safe Zone" spaces to avoid (damage) of the neurovascular bundle. The first wire was placed from posterolateral to anteromedial direction, starting from the fibula; the second, from posteromedial to anterolateral direction; for further stability a third wire in the distal fragment was added. Later on the wires were tensioned at 1400 Newton to increase the stability of the construct and mounted to a semicircular frame. In the area proximal to the fracture site in the anteromedial region, however, three Shanz screws were placed and connected by clamps to the fixator which was blocked once optimal reduction was achieved.

\subsection{Rehabilitation rotocol}


Active and passive rehabilitation was started on the second day after surgery. Limited weight bearing (max8-12 kg) with the aid of Canadian crutches was allowed about 3 weeks after surgery. Partial weight bearing ( $\max 20-30 \mathrm{~kg}$ ) was allowed after assessing the first radiogram, about 30/40 days, and then the progressive/total weight bearing was allowed about 2-3 months after surgery.

\subsection{Human and animal right}

For this type of study any statement relating to studies on humans and animals is not required. Patients gave her informed consent prior to being included in the study. All procedures involving human participants were in accordance with the 1964 Helsinki declaration and its later amendments.

\section{Results}

All patients underwent scheduled clinical and radiographic followups until complete recovery occurred with a variable range from 12 to 24 months, depending on the type of pilon fracture; in fact for the intra-articular fractures, the follow-up to assess the possible onset of tibio-astragalic arthritis was longer, between 24-56 months after surgery.

Dynamization of the external fixator frame was performed, on average at 83.6 days (75-102) after surgery. The fixator was removed about 133.7 days (105-210 days) from surgery.

Recovery from the fracture was confirmed by the presence of a bone callus to XR in at least three cortical (bones) in AP and LL about 160.6 days (120-180) and the clinical evaluation of the absence of pain in the total weight. Radiographic healing of the fractures in 60 patients occurred in 120 days (mean 105 when the external fixator was removed.

The final range of ankle motion was 15 of dorsal flexion and 10 plantar. For 80 cases there was an average score of 83 Mazur (Figure 3).

$$
\text { Mazur score } \quad \text { Closed } ₫ \text { Open }
$$

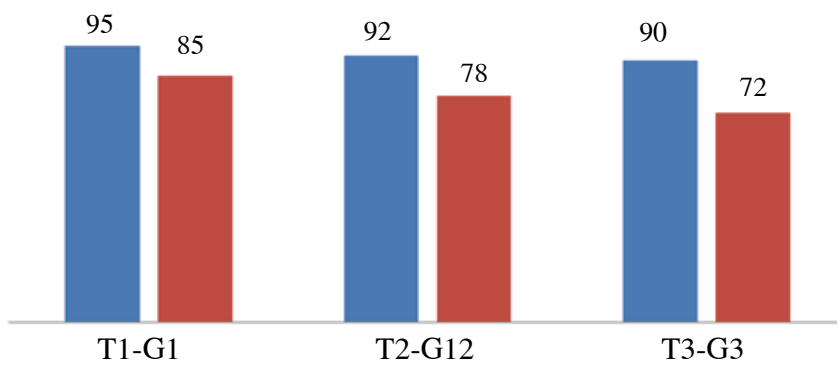

Figure 3. Funtional Outcomes in closed and open fractures according Mazur Score.

For A type fractures the score was 90, while lower values were found in $\mathrm{C}$ type fractures. A mean score of 95 was observed in fractures with soft tissue involvement(Tscherne 1), while 90 in Tscherne 3.In open fractures values varied from 85 (Gustilo 1) to 72 (Gustilo 3).

11 cases $(19.3 \%)$ had skin infection and other early complications; 5 patients $(6 \%)$ developed in nonunions and 5 ankle stiffness.

To the gradual recovery of the bone defect, it was associated the gradual recovery of soft tissue defect and in only for 4 cases it was necessary to perform free grafts of skin (Figure 4, 5, 6, 7).
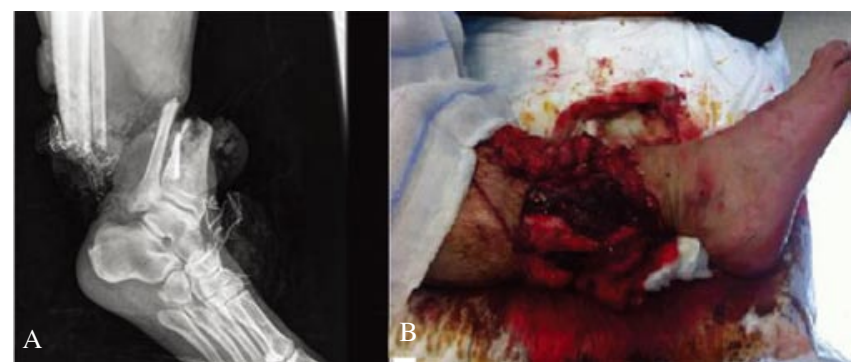

Figure 4. $\mathrm{XR}(\mathrm{A})$ shows the discomposed fractures of tibial pilon and fibula over the tibial-fibula syndesmosis. B shows the open fracture IIIB according Gustilo -Anderson, with a greater soft tissue loss but with posterior tibial vessels and nerves not damage, intact plantar reflex and Mangled Extremity Severity Score $($ MESS $)=5$ point.

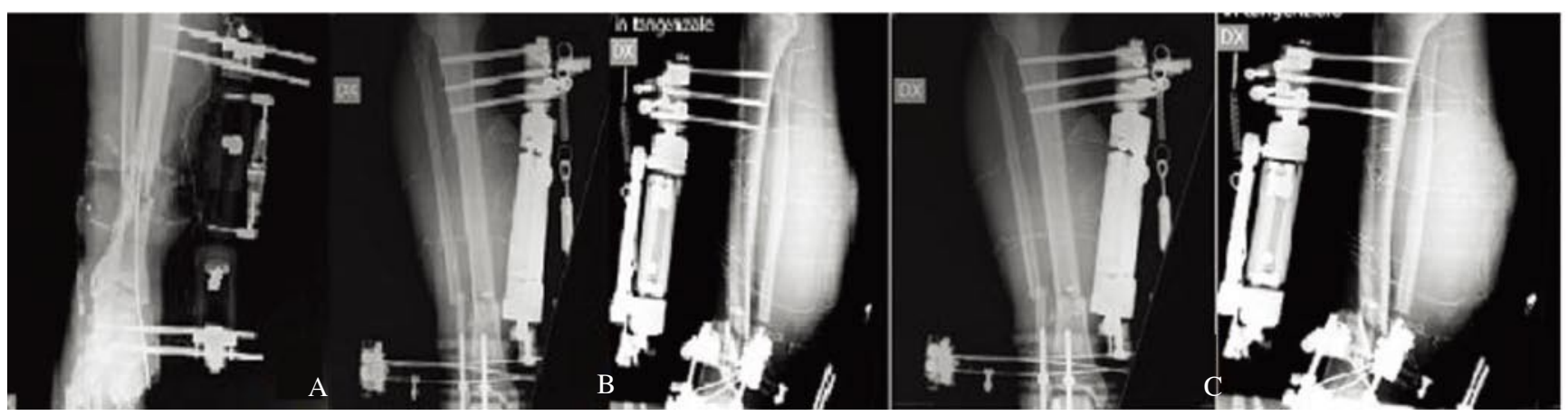

Figure 5. Five treatment in urgency and final of the case.

A shows the treatment in urgency with stabilization of the fracture with external fixator for Monoaxial ligamentotaxis with distal socket and the talus. B shows the schift to an external hybrid fixator Hybrid; C shows the X-ray control always to 2 months with hybrid external fixator. 

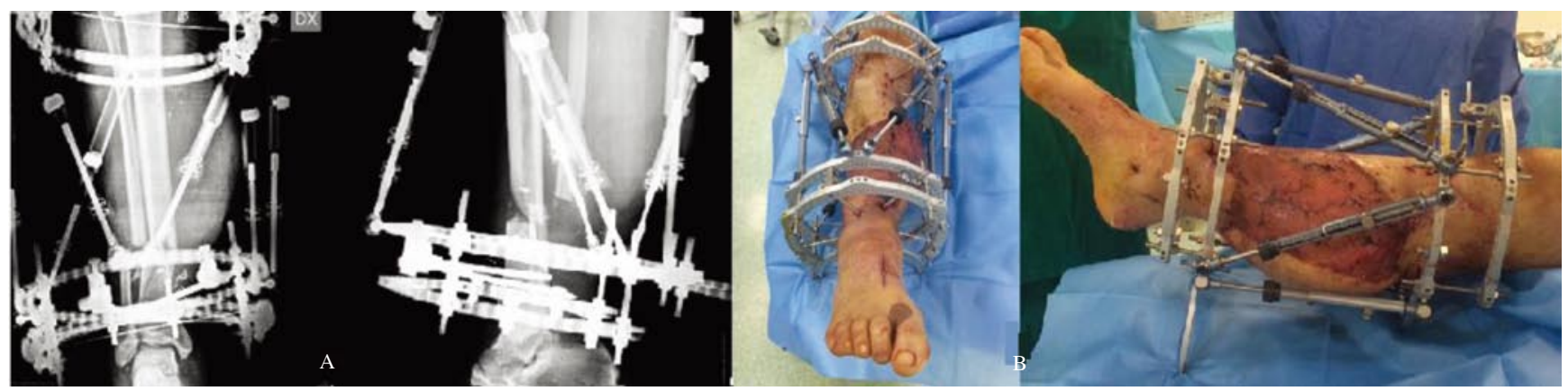

Figure 6. It shows the conclusion of the clinical case with the implantation of an external fixator according Ilizarov (A). B shows perfect soft tissue regeneration after flap quadriceps.

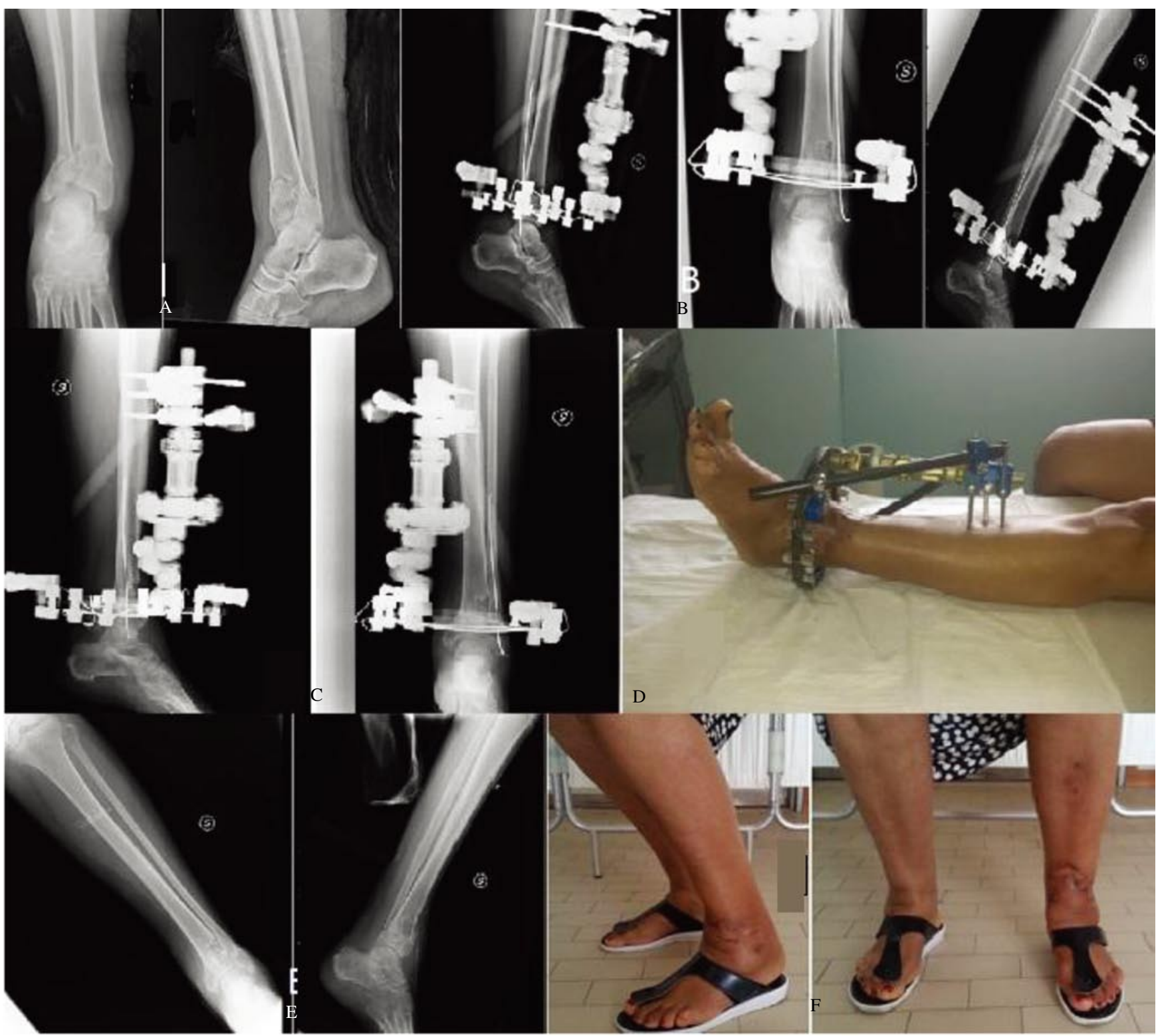

Figure 7. (A) Tibilal pylon fracture type $\mathrm{C} 1$ According AO, in a $55 \mathrm{y} / \mathrm{o}$ woman; (B) Application of hybrid external fixatr and XR control to 70 days; (C) XR Control at 3 months, remove the medial bar and load spillway already granted; (D) At 5th month from the surgery we removed the hybrid external fixator: (E) shows the XR and bone healing; (F) showing removing of months and clinical outcomes. 


\section{Discussion}

Pilon fractures are still a major challenge for the orthopedic surgeon. Their management is guided by the state of the soft tissues, the joint injury and the comminution of the fracture fragments [5,10-14]. The primary objective to be achieved, regardless of the method of synthesis, is to obtain good anatomical reduction of the fracture with the possibility of early weight bearing and avoid more serious consequences [5,10 -14]. In an old study, Tornetta et al [15] analyzed 26 pilon fractures with conventional x-rays and CT scans, observing a $64 \%$ change of the operating procedure when additional information was obtained by using the CT. In fractures with serious damage ofthe soft tissues, metaphyseal comminution and wound exposure, the use of plates and screws is associated with a high failure rate and serious complications, such as infection of the surgical wound and mal-consolidation [12-14]. External fixation is a viable alternative to provide a permanent reduction associated with a decrease of complications [5]. The importance and popularity among orthopedic surgeons of external fixation lies in the fact that the soft tissue is minimally affected and joint movement is preserved, the load can be allowed early, thereby promoting fracture union [5, $10.16-19]$. Although the application of external fixators is a relatively easy procedure, restoration of joint congruity cannot be achieved without minor surgery $[5,10]$. The articular fragments fitted in can usually require the use of bone grafts and additional clamping with k-wires and screws [5,9]. The growing interest in this type of technique has produced, in recent years, two articles on a wide study population[5,19].

The correct timing of treatment is necessary to minimize the risk of complications of the soft tissue $[5,10,19]$. Immediate or early action (within 12-18 hours after trauma) is limited to the stabilization of the tibia and fibula using an external transarticular fixator to preserve the length and obtain a preliminary reduction through ligamentotaxis. The placement of the external fixator creates an optimal environment for the soft tissue healing and the final reconstruction can be performed at a later date [20,21]. Final reconstruction, in fact, can be delayed, until the soft tissue edema decreases, for a period ranging from 7 to 10 days, a time sufficient for the vesicles to heal and to allow a good tolerability to surgery [22]. Some early studies considered ORIF the standard treatment of pilon fractures with good to excellent results between $74 \%$ and $90 \%$ [23]. Recent studies seem to confirm the effectiveness of this method although the fractures taken into consideration presented closed wounds and low soft tissue damage [24]. However Garner et al [25] have shown that a satisfactory anatomical reduction with ORIF of the pilon fractures shown by intraoperative imaging does not correspond to good functional outcomes and quality of life.

The clinical results achieved are comparable to those obtainable with other methods, proving thatthe external fixation is a viable alternative especially when following the instructions [21]. Thehybrid external fixator provides good stability of the fracture with a minimal invasiveness of the soft tissue, thus allowing a more rapid recovery associated with the onset of minor complications and early mobilization. The hospital stay is shorter and this means considerable savings for the health system and, above all, a return for the patient to the daily activities[5,21,26-29].

For more complex fractures and soft tissue involvement, the use of plates and screws has produced a higher incidence of infections and osteomyelitis with a percentage equal to half of the cases treated, $54 \%$ [21]. Frequent complications with ORIF have led to the use of new treatment options: hybrid external fixation $[5,10,19,21]$. The hybrid external fixator has the advantage, over the circular external fixation which gives some rigidity, to have, in the construct ,both a circular and axial locking, so to guarantee sufficient ankle flexibility and create a stable synthesis $[5,10,19,21]$. Our rather large sample of pilon fractures seems to confirm the validity of the management of these fractures with external hybrid fixation, in urgency or delayed, especially those with severe soft tissue involvement and bone exposure; although the classification of the single lesions examined is highly variable. Pilon fractures are often aggravated by complex fracture complications; the treatment with the hybrid external fixator has allowed a stable synthesis with less invasiveness, early weight bearing and good functional recovery.

\section{Authors' contributions}

Giuseppe Rollo, Marco Filipponi, Paolo Pichierri, Valentina Russi, Lorenzo Nalbone, Michele D'arienzo , Sara Cavalera, Gianfranco Corina, Michel Bisaccia and Luigi Meccariello disclose any financial and personal relationships with other people or organisations that could inappropriately influence (bias) their work.

\section{Conflict of interest statement}

All authors disclose any financial and personal relationships with other people or organizations that could inappropriately influence (bias) their work. Examples of potential conflicts of interest include employment, consultancies, stock ownership, honoraria, paid expert testimony, patent applications/registrations, and grants or other funding.

\section{References}

[1]Mauffrey C, Vasario G, Battiston B, Lewis C, Beazley J, Seligson D Tibial pilon fractures: a review of incidence, diagnosis, treatment, and complications. Acta Orthop Belg 2011;77:432-440.

[2]Calori GM, Tagliabue L, Mazza E, de Bellis U, Pierannunzii L, Marelli BM. Tibial pilon fractures: which method of treatment? Injury 2010; 41:1183-1190.

[3]Jacob N., Amin A., Giotakis N., Narayan N., Nayagam S., Trompeter A.J. Management of high-energy tibialpilon fractures. Strat TraumLimbRecon 2015; 10:137-147. 
[4]Cheng W,Li Y, Manyi W. Comparison study of two surgical options for distal tibia fracture-minimally invasive plate osteosynthesis vs. open reduction and internal fixation. International Orthopaedics (SICOT) 2011; 35:737-742.

[5]Falzarano G, Medici A, Grubor P, Grubor M, Meccariello L. Emergent hybrid external fixation for tibial pilon fractures in adults. J Acute Dis 2015; 4(4): 322-325.

[6]Gustilo RB, Anderson JT. Prevention of infection in the treatment of one thousand and twenty-five open fractures of long bones: retrospective and prospective analyses. J Bone Joint Surg Am 1976;58:453-458.

[7]Tscherne H. Pathophysiology and classification of soft tissue injuries associated with fractures. In: Tscherne H, Gotzen L, editors. Fractures with soft tissue injuries. Berlin: Springer-Verlag; 1984, p. 6-7.

[8]Modhia UM, Dickens AJ, Glezos CD, Gehlert RJ and DeCoster TA. Under-Utilization of the OTA Fracture Classification in the Orthopaedic Trauma Literature. Iowa Orthop J 2014; 34: 50-54.

[9]Kodama N, Takemura Y, Shioji S, Imai S. Arthrodesis of the ankle using an anterior sliding tibial graft for osteoarthritis secondary to osteonecrosis of the talus: A comparison of vascularised non-vascularised grafts. Bone Joint J 2016;98-B(3):359-64. doi: 10.1302/0301-620X.98B3.36154.

[10]Milenkovi S, Mitkovi M, Mici I, Mladenovi D, Najman S, Trajanovi M, Mani M, Mitkovi M. Distal tibialpilon fractures (AO/OTA type B, and $\mathrm{C}$ ) treated with the external skeletal and minimal internal fixation method. Vojnosanit Pregl 2013 ;70(9):836-41.

[11]Falzarano G, Medici A, Nobile F, RobertoVL, Viglione S, Errico G, Franzese R, Falzarano A, Pavone M, Meccariello L. The advantage/ benefit of the mippo method in emergency treatmentsof type a tibial pilon fractures in vascular limb diseases: a case report. Canadian Open Orthopaedics and Traumatol J 2015;2(2):1-6.

[12]Vidovic D, Matejcic'A, Ivica M, Jurisic' D, Elabjer E, Bakota B. Minimally-invasive plate osteosynthesis in distal tibial fractures: Results and complications. Injury, Int J Care Injured 2015; 46S: S96-S99.

[13]Tang X, Liu L, Tu CQ, Li J, Li Q, Pei FX. Comparison of early and delayed open reduction and internal fixation for treating closed tibial pilon fractures. Foot Ankle Int 2014;35:657-664.

[14]Cisneros LN, Gómez M, Alvarez C, Millán A, De Caso J, Soria L. Comparision of outcomes of tibial plafond fracture managed by hybrid external fixation versus two-stage management whit final plate fixation. Indian J Orthop 2016;50(2):123-130.

[15]Tornetta 3rd P, Weiner L, Bergman M, Watnik N, Steuer J, Kelley M. Pilon fractures: treatment with combined internal and external fixation. $J$ Orthop Trauma 1993;7:489-496.

[16]Babis G.C, Kontovazenitis P, Evangelopoulos D.S, Tsailas P, Nikolopoulos K, Soucacos P.N. Distal tibial fractures treated with hybrid external fixation. Injury, Int J Care Injured 2010;41 :253-258.
[17]Lali I, Daraboš N, Stankovi M, Gojkovi Z, Obradovi M, Mari D Treatment of complex tibial plateau fractures using Ilizarov technique. Acta Clin Croat 2014 ;53(4):437-448.

[18]Saghieh S. Distal tibia fracture: non bridging hybrid external fixator. In: Weinstein SL, Hoballah JJ, Saghieh S, editors. Operative dictations in orthopedic surgery. New York: Springer; 2013, p. 227-230.

[19]Galante VN, Vicenti G, Corina G, Mori C, Abate A, Picca G, Conserva V, Speciale D, Scialpi L, Tartaglia N, Caiaffa V, Moretti B. Hybrid external fixation in the treatment of tibial pilon fractures: A retrospective analysis of 162 fractures. Injury 2016 ;47(4):S131-S137. doi: 10.1016/ j.injury.2016.07.045.

[20]Luo H, Chen L, Liu K, Peng S, Zhang J, Yi Y. Minimally invasive treatment of tibial pilon fractures through arthroscopy and external fixator-assisted reduction. Springerplus 2016;5(1):1923.

[21]Meng YC, Zhou XH. External fixation versus open reduction and interna fixation for tibial pilon fractures: A meta- on observational studies. Chin J Traumatol 2016;19(5):278-282.

[22]Pollak AN, McCarthy ML, Bess RS, Agel J, Swiontkowski MF. Outcomes after treatment of high-energy tibial plafond fractures. $J$ Bone Joint Surg Am 2003;85(10):1893-1900.

[23]Rüedi T. Fractures of the lower end of the tibia into the ankle joint: results 9 years after open reduction and internal fixation. Injury 1973;5:130-134.

[24]Grose A, Gardner MJ, Hettrich C, Fishman F, Lorich DG, Asprinio DE. Open reduction and internal fixation of tibialpilon fractures using a lateral approach. J Orthop Trauma 2007;21:530-7.

[25]Garner MR, Fabricant PD, Schottel PC, Berkes MB, Shaffer AD, Ni A, Lorich DG. Standard perioperative imaging modalities are unreliable in assessing articular congruity of ankle fractures. J Orthop Trauma 2015;29(4):e161-5. doi: 10.1097/BOT.0000000000000219.

[26]Watson JT, Moed BR, Karges DE, Cramer KE. Pilon fractures. Treatment protocol based on severity of soft tissue injury. Clin Orthop Relat Res 2000;375:78-90.

[27]Danoff JR, Saifi C, Goodspeed DC, Reid JS. Outcome of 28 open pilon fractures with injury severity-based fixation. Eur J Orthop Surg Traumatol 2015;25(3):569-575.

[28]Crawford B, Watson JT, Jackman J, Fissel B, Karges DE. End-stage hindfoot arthrosis: outcomes of tibiocalcaneal fusion using internal and Ilizarov fixation. J Foot Ankle Surg 2014;53(5):609-914.

[29]Piscitelli L, Bisaccia M, Meccariello L, Falzarano G, Medici A, Maiettini M, Rebonato A, Rinonapoli G, Caraffa A. The emergency and delay management in total talus extrusion: Case report and review of literature after 24 months of follow up. J Acute Dis 2016; 5(4):169-72. Doi: 10.1016/j.joad.2016.04.001 